\title{
Relationship between the Biofilm-Forming Capacity and Antimicrobial Resistance in Clinical Acinetobacter baumannii Isolates: Results from a Laboratory-Based In Vitro Study
}

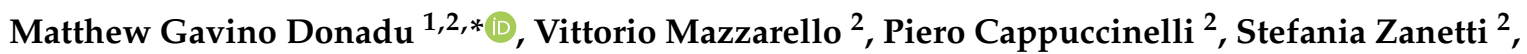 \\ Melinda Madléna ${ }^{3}$, Ádám László Nagy ${ }^{4}$, Anette Stájer ${ }^{5}$, Katalin Burián ${ }^{6}$ (D) and Márió Gajdács ${ }^{3,7}$ \\ 1 Hospital Pharmacy, Azienda Ospedaliero Universitaria di Sassari, 07100 Sassari, Italy \\ 2 Department of Biomedical Sciences, University of Sassari, Sassari, 07100 Sassari, Italy; \\ vmazza@uniss.it (V.M.); pcappuc@gmail.com (P.C.); zanettis@uniss.it (S.Z.) \\ 3 Department of Oral Biology and Experimental Dental Research, Faculty of Dentistry, University of Szeged, \\ Tisza Lajos körút 63, 6720 Szeged, Hungary; madlenamelinda784@gmail.com (M.M.); \\ mariopharma92@gmail.com (M.G.) \\ 4 Department of Prosthodontics, Faculty of Dentistry, University of Szeged, Tisza Lajos körút 62-64, \\ 6720 Szeged, Hungary; nagy.adam.laszlo@stoma.szote.u-szeged.hu \\ 5 Department of Periodontology, Faculty of Dentistry, University of Szeged, Tisza Lajos körút 62-64, \\ 6720 Szeged, Hungary; stajeranette@gmail.com \\ check for \\ updates \\ Citation: Donadu, M.G.; Mazzarello, \\ V.; Cappuccinelli, P.; Zanetti, S.; \\ 6 Department of Medical Microbiology, Albert Szent-Györgyi Health Center and Faculty of Medicine, \\ University of Szeged, Semmelweis Utca 6, 6725 Szeged, Hungary; burian.katalin@med.u-szeged.hu \\ 7 Faculty of Medicine, Institute of Medical Microbiology, Semmelweis University, Nagyvárad tér 4, \\ 1089 Budapest, Hungary \\ * Correspondence: mdonadu@uniss.it
} Madléna, M.; Nagy, Á.L.; Stájer, A.; Burián, K.; Gajdács, M. Relationship between the Biofilm-Forming Capacity and Antimicrobial

Resistance in Clinical Acinetobacter baumannii Isolates: Results from a Laboratory-Based In Vitro Study. Microorganisms 2021, 9, 2384. https://doi.org/10.3390/ microorganisms 9112384

Academic Editor: Akikazu Sakudo

Received: 9 October 2021

Accepted: 17 November 2021

Published: 18 November 2021

Publisher's Note: MDPI stays neutral with regard to jurisdictional claims in published maps and institutional affiliations.

Copyright: (C) 2021 by the authors Licensee MDPI, Basel, Switzerland. This article is an open access article distributed under the terms and conditions of the Creative Commons Attribution (CC BY) license (https:/ / creativecommons.org/licenses/by/ $4.0 /)$.

\begin{abstract}
The relationship between the multidrug-resistant (MDR) phenotype and biofilm-forming capacity has been a topic of extensive interest among biomedical scientists, as these two factors may have significant influence on the outcomes of infections. The aim of the present study was to establish a possible relationship between biofilm-forming capacity and the antibiotic-resistant phenotype in clinical Acinetobacter baumannii (A. baumannii) isolates. A total of $n=309$ isolates were included in this study. Antimicrobial susceptibility testing and the phenotypic detection of resistance determinants were carried out. The capacity of isolates to produce biofilms was assessed using a crystal violet microtiter-plate-based method. Resistance rates were highest for ciprofloxacin $(71.19 \% ; \mathrm{n}=220)$, levofloxacin ( $n=68.61 \% ; n=212)$, and trimethoprim-sulfamethoxazole ( $n=66.02 \% ; n=209) ; 42.72 \%$ $(n=132)$ of isolates were classified as MDR; $22.65 \%(n=70)$ of tested isolates were positive in the modified Hodge-test; the overexpression of efflux pumps had significant effects on the susceptibilities of meropenem, gentamicin, and ciprofloxacin in $14.24 \%(n=44), 6.05 \%(n=19)$, and $27.51 \%(n=85)$, respectively; $9.39 \%(n=29), 12.29 \%(n=38), 22.97 \%(n=71)$, and $55.35 \%(n=170)$ of isolates were non-biofilm-producing and weak, moderate, and strong biofilm producers, respectively. A numerical, but statistically not significant, difference was identified between the MDR and non-MDR isolates regarding their biofilm-forming capacity (MDR: $0.495 \pm 0.309$ vs. non-MDR: $0.545 \pm 0.283 ; p=0.072$ ), and no association was seen between resistance to individual antibiotics and biofilm formation. Based on numerical trends, MER-resistant isolates were the strongest biofilm producers $(p=0.067)$. Our study emphasizes the need for additional experiments to assess the role biofilms have in the pathogenesis of A. baumannii infections.
\end{abstract}

Keywords: Acinetobacter baumannii; antibiotic resistance; multidrug resistance; MDR; biofilm; crystal violet; phenotypic assay

\section{Introduction}

Due to the growing number of patients affected by invasive medical interventions and immunosuppression, the prevalence and disease burden of infections caused by nonfer- 
menting Gram-negative bacteria (NGFNBs; including Pseudomonas aeruginosa, Acinetobacter spp., and Stenotrophomonas maltophilia) have also increased considerably [1]. The genus Acinetobacter is an oxidase-negative coccobacillus, consisting of $>40$ genospecies, among which the Acinetobacter baumannii-calcoaceticus complex is the most clinically relevant [2]. A. baumannii is an important opportunistic pathogen in nosocomial infections, which-although traditionally considered a low-grade pathogen-has been implicated in a wide array of infectious syndromes, including bacteremia, pneumonia (especially ventilator-associated pneumonia (VAP)), urinary tract infections, and eye and wound infections, affecting patients with immunosuppression, severe underlying conditions, or patients in critical condition (treated in Intensive Care Units (ICUs)) [3-6]. Patients experiencing prolonged ( $\geq 90$ days) hospitalization and previous antimicrobial therapy are particularly at risk [7]. The reported mortality rate of invasive $A$. baumannii infections is considerably high, ranging between $23 \%$ and $68 \%$ and between $0 \%$ and $64 \%$ for nosocomial and community-associated infections, respectively [8]. In a recent meta-analysis of 114 studies, the estimated mortality of A. baumannii VAP has been reported at $42.6 \%$ (95\% CI, 37.2-48.1\%), while other studies have shown that the mortality rate of the A. baumannii bacteremia in ICUs was between $37 \%$ and $52 \%$, while for VAP, this figure might be as high as $84 \%$ [9].

Since the 2000s, the emergence of multidrug-resistant (MDR) and extensively drugresistant (XDR) strains of $A$. baumannii has become a critical concern for healthcare professionals worldwide, with an alarmingly low number of antibiotics left useful for their treatment [10]. The previously mentioned meta-analysis reported the prevalence of MDR A. baumannii in nosocomial pneumonia at 79.9\% (95\% CI, 73.9-85.4\%) in some regions (Central America, Latin America, and the Caribbean), where all isolates are now considered MDR [9]. In addition to their nonsusceptibility to many commonly used antimicrobials, Acinetobacter spp. are characterized by a considerable genomic plasticity, and a tremendous capacity to uptake novel resistance determinants (via plasmids, transposons, and integrons, accumulating them in large genomic islands in their chromosome), leading to infections where they are no longer a safe and effective drug for treatment [11]. Carbapenem-resistant A. baumannii (CR-AB) has been designated as a "critical priority" pathogen for the development of novel antibiotics and anti-infectives, according to the report published by the World Health Organization (WHO) [12].

Biofilms are complex assemblages of bacteria and an external matrix material, consisting of exopolysaccharide (EPS) and other carbohydrates, proteins, lipids, environmental DNA (eDNA), ions, and water $[13,14]$. Biofilms allow for a protective form of growth and metabolic inactivity for microorganisms, providing a safe haven against environmental stressors (sheer forces, heat and drying damage), attacks of the immune system (phagocytes, complement), disinfectants, and antimicrobials [15]. In fact, bacteria embedded in deeper layers of the biofilm seldom come into contact with antibiotics, due to the inability of these drugs to adequately penetrate into its deeper layers; this results in 10-1000-fold higher minimum inhibitory concentration (MIC) values, compared to the ones observed for planktonic cells [16]. The biofilm-forming capacity of $A$. baumannii largely contributes to its success as a nosocomial pathogen, as it allows for survival within hospital environments (e.g., taps and fluid containers) and on vascular catheters or other implantable devices [17]. It has been described that $A$. baumannii may survive for 4-5 months on abiotic surfaces, while so-called "hypervirulent" forms of $A$. baumannii were also associated with strong biofilm production [16,17]. The relationship between the antimicrobial-resistant phenotype and the biofilm-forming capacity has been a topic of extensive interest among biomedical scientists, as these two factors may have significant influence on the outcomes of infections $[18,19]$. In addition, there have been studies showing that nonlethal concentrations of antibiotics induced biofilm production in various Gram-positive and Gram-negative bacteria, suggesting a common response to overcome external stressors [20]. The aim of the present study was to establish a possible relationship between biofilm-forming capacity and the antibiotic-resistant phenotype in clinical $A$. baumannii isolates. 


\section{Materials and Methods}

\subsection{Collection of Isolates}

A total of three hundred and nine $(\mathrm{n}=309)$ A. baumannii isolates were included in this study, which were kindly provided from the strain collections of a Hungarian and Italian tertiary-care hospital. The study used a cross-sectional design, with microorganisms that were isolated between 1 January 2019 and 1 January 2020, originating from various types of clinical specimens, being randomly selected to be included in our experiments. During the study, A. baumannii clinical isolate no. 59738 (MDR isolate, weak biofilm producer) and $A$. baumannii ATCC 19606 (susceptible isolate, strong biofilm producer) were used as control strains (the latter was obtained from the American Type Culture Collection, Manassas, VI, USA) [21]. Stock cultures were stored at $-80{ }^{\circ} \mathrm{C}$ in a cryopreservation medium $(700 \mu \mathrm{L}$ of trypticase soy broth $+300 \mu \mathrm{L}$ of $50 \%$ glycerol) until use.

\subsection{Re-Identification of Isolates}

All isolates included in our study were re-identified as A. baumannii before further experiments. Re-identification of isolates was carried out using matrix-assisted laser desorption/ionization-time-of-flight mass spectrometry (MALDI-TOF MS; Bruker Daltonics, Bremen, Germany); to perform the MALDI-TOF assay, bacterial cells from fresh overnight cultures were transferred to a stainless-steel target. An on-target extraction was performed by adding $1 \mu \mathrm{L}$ of $70 \%$ formic acid prior to the matrix. After drying at room temperature, the cells were covered with $1 \mu \mathrm{L}$ of matrix ( $\alpha$-cyano- 4 -hydroxy cinnamic acid in 50\% acetonitrile/2.5\% trifluoro-acetic acid; Bruker Daltonics, Bremen, Germany). Mass spectrometry analyses were performed by a MicroFlex MALDI Biotyper (Bruker Daltonics, Bremen, Germany) in positive linear mode across the $\mathrm{m} / \mathrm{z}$ range from 2 to $20 \mathrm{kDa}$; for each spectrum, 240 laser shots at $60 \mathrm{~Hz}$ in groups of 40 shots per sampling area were collected [22]. For analyses of spectra, the MALDI Biotyper RTC 3.1 software and the MALDI Biotyper Library 3.1 (Bruker Daltonics. Bremen, Germany) were utilized. After analysis, a $\log$ (score) value was assigned to all isolates, indicating the reliability of MALDI-TOF MS identification. The $\log$ (score) values were evaluated as follows: a $\log$ (score) $<1.69$ showed unreliable identification, 1.70-1.99 corresponded to probable genus-level identification, 2.00-2.29 corresponded to reliable genus-level identification, while a score $\geq 2.30$ corresponded to reliable species-level identification [23].

\subsection{Antimicrobial Susceptibility Testing and Resistotyping}

Antimicrobial susceptibility testing for respective isolates was carried out using the Kirby-Bauer disk diffusion method (Oxoid, Basingstoke, UK), and in subsequent experiments (when relevant) with E-tests (Liofilchem, Roseto degli Abruzzi, Italy) on Mueller-Hinton agar (MHA) (bioMérieux, Marcy-l'Étoile, France) plates in the case of imipenem (IMI; $10 \mu \mathrm{g}$ ), meropenem (MER; $10 \mu \mathrm{g})$, ciprofloxacin (CIP; $5 \mu \mathrm{g}$ ), levofloxacin (LEV; $5 \mu \mathrm{g}$ ), trimethoprim-sulfamethoxazole (SXT; 23.75/1.25 $\mu \mathrm{g}$ ), gentamicin (GEN; $10 \mu \mathrm{g})$, and amikacin (AMI; $30 \mu \mathrm{g}$ ). Tigecycline (TIG) susceptibility was performed using E-tests on MHA, while colistin (COL) susceptibility was performed using the broth microdilution method in cation-adjusted Mueller-Hinton broth (MERLIN Diagnostika GmbH, Bremen, Germany). The isolates were grouped into distinct resistotypes based on the presence of phenotypic resistance to relevant antimicrobials [24]. Interpretation of the results was based on the recommendations of the European Committee on Antimicrobial Susceptibility Testing (EUCAST) relevant at the time of isolation, with the exception of TIG, where the U.S. Food and Drug Administration (FDA) breakpoint (resistance of MIC $>4 \mathrm{mg} / \mathrm{L}$ ) was applied [25,26]. Intermediate results were grouped with and reported as resistant [22]. Classification of the isolates as (MDR; resistance to at least one agent in $\geq 3$ antibiotic groups) was based on Magiorakos et al. [27]; in subsequent analyses, isolates were divided into non-MDR and MDR. A multiple-antibiotic-resistance (MAR) index-ranging between 0 and 1-was calculated by dividing the total number of detected resistance to antimicrobials for each isolate by the total number of tested antimicrobials [24]. 


\subsection{Phenotypic Detection of Carbapenemase and Metallo- $\beta$-Lactamase Production}

To establish carbapenemase production in the A. baummanni included in the study, the isolates were subjected to the modified Hodge (cloverleaf) test, as previously described [28]. In the assay, MER disks (10 $\mu \mathrm{g}$; Oxoid, Basingstoke, UK) were utilized and Escherichia coli ATCC 25922 was used as an indicator organism. Metallo- $\beta$-lactamase (MBL) production was tested using the imipenem/EDTA combined disk test (CDT), as described previously [29]. In preparation to this assay, imipenem/EDTA disks were prepared by adding $750 \mu \mathrm{g}$ of a sterile $0.5 \mathrm{M}$ EDTA solution to a $10 \mu \mathrm{g}$ imipenem disk, and then disks were dried in a $37^{\circ} \mathrm{C}$ incubator. Disks containing EDTA alone served as a negative control. The isolate was considered positive for MBLs if the inhibition zone diameter of the imipenem/EDTA disk increased by $\geq 7 \mathrm{~mm}$ compared to the imipenem disk alone [28].

\subsection{Phenotypic Detection of Bacterial Efflux Pumps Contributing to the MDR Phenotype}

To establish the effects of the overexpression of resistance-nodulation-division (RND) efflux pumps on the susceptibility of tested antimicrobials, a phenylalanine-arginine $\beta$ naphthylamide $(\mathrm{PA} \beta \mathrm{N})$ plate-based assay was performed, as described by Salehi et al. [30]. During the experiments, the concentration of PA $\beta N$ (Sigma-Aldrich, St. Louis, MO, USA) — a compound with well-known efflux pump inhibitory activity-was $40 \mu \mathrm{g} / \mathrm{mL}$ in the agar base, while CIP, GEN, and MER were selected as test antimicrobials; a four-fold decrease in the MICs (determined by E-tests; Liofilchem, Roseto degli Abruzzi, Italy) of the antimicrobials in the presence of PA $\beta \mathrm{N}$, compared to the MIC values without the inhibitor, was considered as positivity for efflux pump overexpression [30].

\subsection{Biofilm Formation Assay}

The capacity of respective $A$. baumannii isolates to produce biofilms was assessed using a microtiter-plate-based method previously described by Ramos-Vivas et al. [31]. In brief, fresh A. baumannii cultures (grown on Luria-Bertani (LB) agar) were inoculated into $5 \mathrm{~mL}$ of Luria-Bertani (LB) broth and incubated overnight at $37^{\circ} \mathrm{C}$. The following day, $180 \mu \mathrm{L}$ of LB broth and $20 \mu \mathrm{L}$ of $A$. baumannii suspension (set at $10^{6} \mathrm{CFU} / \mathrm{mL}$ ) were measured onto 96-well flat-bottomed microtiter plates, to a final volume of $200 \mu \mathrm{L}$, and incubated for $24 \mathrm{~h}$ at $37^{\circ} \mathrm{C}$. After the incubation period, the supernatants were discarded and the wells were washed three times using $200 \mu \mathrm{L}$ of phosphate-buffered saline (PBS; pH at 7.2), to remove planktonic cells, which may interfere with the interpretation of the results. After washing, the wells were fixed with $250 \mu \mathrm{L}$ of methanol (Sigma-Aldrich, St. Louis, MO, USA) for 10 min and stained with a 1.0\% crystal violet (CV; Sigma-Aldrich, St. Louis, MO, USA) solution for $15 \mathrm{~min}$. Subsequently, the CV solution was discarded, and the wells were washed three times with purified water, to remove excess stains. The contents of the wells were re-suspended in $250 \mu \mathrm{L}$ of $33 \% \mathrm{~V} / \mathrm{V} \%$ glacial acetic acid (Sigma-Aldrich, St. Louis, MO, USA), and the absorbance at $570 \mathrm{~nm}\left(\mathrm{OD}_{570}\right)$ was measured using a microtiter plate reader. All experiments were carried out in triplicate. The $\mathrm{OD}_{570}$ values of the measurements were recorded as the mean \pm standard deviation (SD). The interpretation of the results was carried out based on the recommendations of Stepanovic et al. [32], namely, a cut-off value of optical density $\left(\mathrm{OD}_{\mathrm{c}}\right)$ was calculated using the following formula: $\mathrm{OD}_{\mathrm{c}}=$ average $\mathrm{OD}$ of the negative control $+(3 \times$ standard deviations of negative control $)$. Subsequently, isolates were classified into the following categories, based on their $\mathrm{OD}_{570}$ measurements: strong biofilm producer $(\mathrm{OD}>4 \times \mathrm{ODc})$; medium biofilm producer $(4 \times \mathrm{ODc} \geq \mathrm{OD}>2 \times \mathrm{ODc})$; weak biofilm producer $(2 \times \mathrm{ODc} \geq \mathrm{OD}>\mathrm{ODc})$; and nonbiofilm producer $(\mathrm{OD} \leq \mathrm{ODc})[32]$.

\subsection{Statistical Analysis}

Descriptive statistical analysis (including means with ranges and percentages to characterize data) was performed using Microsoft Excel 2013 (Redmond, WA, USA, Microsoft Corp.). The normality of variables was tested using the Kolmogorov-Smirnov test. Independent sample $t$-tests were performed to compare measurements of $\mathrm{OD}_{570}$ (for biofilm production) between susceptible/resistant isolates to individual antibiotics, and among 
MDR and non-MDR A. baumannii isolates, respectively. Comparisons of groups $\left(\mathrm{OD}_{570}\right.$ values for isolates resistant to respective antibiotics) were made by one-way analysis of variance (ANOVA) with Tukey's post hoc tests. Statistical analyses were performed with SPSS software version 22 (IBM Corp., New York, NY, USA).

\subsection{Ethical Considerations}

The study was conducted in accordance with the Declaration of Helsinki and national and institutional ethical standards. Ethical approval for the study protocol was obtained from the Human Institutional and Regional Biomedical Research Ethics Committee, University of Szeged (registration number: 140/2021-SZTE [5019]).

\section{Results}

\subsection{Antibiotic Susceptibility of Isolates Included in the Study}

After re-identification with MALDI-TOF MS, all three hundred and nine $(\mathrm{n}=309)$ isolates were verified as $A$. baumannii, and thus, they were included in the subsequent experiments. The antimicrobial resistance levels of the $A$. baumannii isolates included in the study were the following (in decreasing order): CIP 71.19\% $(\mathrm{n}=220), \mathrm{LEV} 68.61 \%$ $(\mathrm{n}=212)$, SXT 66.02\% $(\mathrm{n}=209)$, GEN 60.84\% $(\mathrm{n}=188)$, IMI 52.75\% $(\mathrm{n}=163)$, MER 51.46\% $(\mathrm{n}=159)$, AMI 38.51\% $(\mathrm{n}=119)$, TIG 38.51\% $(\mathrm{n}=44 ; \mathrm{MIC}>4 \mathrm{mg} / \mathrm{L})$, and COL $2.27 \%(\mathrm{n}=7$; MIC $>2 \mathrm{mg} / \mathrm{L})$; overall, $42.72 \%(\mathrm{n}=132)$ of the isolates were classified as MDR, based on the criteria of Magiorakos et al. The distribution of the various resistotypes detected among A. baumannii isolates is presented in Table 1: twenty-one (I-XXI) different resistotypes were identified, with the most numerous being resistotype XVIII (resistant to CIP, LEV, IMI, MER, GEN, AMI, and SXT; 15.21\%), XIV (resistant to CIP, LEV, GEN, AMI, and SXT; 9.40\%), and IV (resistant to GEN and SXT; 9.06\%).

Table 1. Resistotype distribution and MAR indices of respective isolates.

\begin{tabular}{|c|c|c|c|c|}
\hline Resistotypes & Resistance Pattern & MAR Index & MDR Status * & $\begin{array}{c}\text { Ratio of Isolates } \\
(\mathrm{n}, \%)\end{array}$ \\
\hline I & $C I P$ & 0.111 & \multirow{13}{*}{ non-MDR $(57.28 \% ; \mathrm{n}=177)$} & $9(2.91 \%)$ \\
\hline II & $L E V$ & 0.111 & & $10(3.24 \%)$ \\
\hline III & SXT & 0.111 & & $20(6.47 \%)$ \\
\hline IV & GEN, SXT & 0.222 & & $28(9.06 \%)$ \\
\hline $\mathrm{V}$ & $C I P, S X T$ & 0.222 & & $3(0.97 \%)$ \\
\hline VI & $C I P, L E V$ & 0.222 & & $14(4.53 \%)$ \\
\hline VII & $I M I, M E R, S X T$ & 0.333 & & $12(3.88 \%)$ \\
\hline VIII & $I M I, M E R, G E N$ & 0.333 & & $18(5.83 \%)$ \\
\hline IX & $C I P, L E V, S X T$ & 0.333 & & $18(5.83 \%)$ \\
\hline$x$ & $C I P, L E V, M E R$ & 0.333 & & $6(1.94 \%)$ \\
\hline XI & $C I P, L E V, I M I$ & 0.333 & & $10(3.24 \%)$ \\
\hline XII & $C I P, L E V, G E N$ & 0.333 & & $9(2.91 \%)$ \\
\hline XIII & $C I P, L E V, I M I, M E R$ & 0.444 & & $20(6.47 \%)$ \\
\hline XIV & CIP, LEV , GEN, AMI, SXT & 0.555 & \multirow{8}{*}{$\operatorname{MDR}(42.72 \% ; \mathrm{n}=132)$} & $29(9.40 \%)$ \\
\hline $\mathrm{XV}$ & CIP , LEV , GEN, AMI, SXT, TIG & 0.666 & & $20(6.47 \%)$ \\
\hline XVI & CIP, LEV IMI, MER, GEN, SXT & 0.666 & & $12(3.88 \%)$ \\
\hline XVII & CIP , LEV , IMI, MER, GEN, TIG & 0.666 & & $10(3.24 \%)$ \\
\hline XVIII & CIP , LEV IMI, MER, GEN, AMI, SXT & 0.777 & & $47(15.21 \%)$ \\
\hline XIX & CIP , LEV , IMI, MER, GEN, AMI, SXT, TIG & 0.888 & & $7(2.27 \%)$ \\
\hline$X X$ & CIP , LEV , IMI, MER, GEN, AMI, SXT, COL & 0.888 & & $5(1.62 \%)$ \\
\hline XXI & CIP , LEV , IMI, MER, GEN, AMI, SXT, TIG, COL & 1.000 & & $2(0.65 \%)$ \\
\hline
\end{tabular}

* based on the criteria of Magiorakos et al. [27]. 


\subsection{Detection of Carbapenemase-Production and Efflux Pump-Overexpression Using Phenotypic Methods}

To identify the contribution of various resistance mechanisms in the drug resistance of the relevant $A$. baumannii isolates, phenotypic tests were utilized. The modified Hodge test (MHT) was used to detect for the production of carbapenemases: overall, 22.65\% $(n=70)$ of tested isolates (i.e., $42.94 \%$ of isolates resistant to either IMI, MER, or both) were positive for the phenotypic detection of carbapenemases in the MHT assay. MBL production was observed in $9.06 \%(n=28)$ of the tested isolates (i.e., $17.18 \%$ of isolates resistant to either IMI, MER, or both) using the imipenem/EDTA combined disk test (CDT). Overexpression of the RND efflux pumps (based on the results of the Pa $\beta N$ screening agar) had significant effects on the MICs of MER in $14.24 \%$ ( $n=44$; i.e., $27.67 \%$ of isolates resistant to MER), while this was $6.05 \%$ in the case of GEN ( $n=19$; i.e., $10.11 \%$ of isolates resistant to GEN) and $27.51 \%$ in the case of CIP ( $n=85$; i.e., $38.64 \%$ of isolates resistant to CIP), which was demonstrated by the four-fold decrease in MICs detected in the presence of the efflux inhibitor compound. In the case of $n=17$ and $n=8$ isolates, simultaneous positivity of the efflux pump-overexpression-MHT test and efflux pump-overexpression-CDT test was detected, respectively.

\subsection{Biofilm Formation among $M D R$ and Non-MDR A. baumannii Isolates}

Biofilm formation assays were carried out in a microtiter plate-based platform, using $\mathrm{CV}$ staining. The $\mathrm{OD}_{570}$ values for the negative control (clinical isolate no. 59738) and the positive control (ATCC 19606) were $0.091 \pm 0.008$ and $0.388 \pm 0.051$, respectively. Therefore, the Odc value was set at 0.115 , and the classification breakpoints were the following: nonbiofilm producer: $\mathrm{OD} \leq 0.115$, weak biofilm producer: $0.230 \geq \mathrm{OD}>0.115$, medium biofilm producer: $0.460 \geq \mathrm{OD}>0.230$, and strong biofilm producer: $\mathrm{OD}>0.460$. Based on this, $9.39 \%(n=29), 12.29 \%(n=38), 22.97 \%(n=71)$, and $55.35 \%(n=170)$ were non-biofilm producers, and weak, moderate, and strong biofilm producers, respectively; $\mathrm{OD}_{570}$ values ranged between $0.022 \pm 0.003$ and $1.192 \pm 0.086$, with the median value being $0.501(0.466$, 0.534). In addition, $62.14 \%(\mathrm{n}=192)$ of isolates were more potent biofilm producers than the ATCC 19606 strain. A numerical but statistically not significant difference was identified between the MDR and non-MDR isolates regarding their biofilm-forming capacity overall (MDR: $0.495 \pm 0.309$ vs. non-MDR: $0.545 \pm 0.283 ; p=0.072$ ) (Figure 1). During the analysis for individual associations between antibiotic resistance and biofilm propensity (where COL was excluded, due to the low number of resistant isolates), concordance was seen with the overall results, as no significant differences were shown for CIP (susceptible [S]: $0.556 \pm 0.329$ vs. resistant [R]: $0.499 \pm 0.308 ; p=0.55$ ), LEV (S: $0.523 \pm 0.299$ vs. R: $0.508 \pm 0.276 ; p=0.63$ ), SXT (S: $0.555 \pm 0.262$ vs. R: $0.492 \pm 0.306 ; p=0.28$ ), GEN (S: $0.537 \pm 0.329$ vs. R: $0.463 \pm 0.302 ; p=0.66$ ), AMI (S: $0.560 \pm 0.283$ vs. R: $0.481 \pm 0.330$; $p=0.99$ ), TIG (S: $0.559 \pm 0.349$ vs. R: $0.471 \pm 0.279 ; p=0.81$ ), IMI (S: $0.605 \pm 0.283$ vs. R: $0.538 \pm 0.331 ; p=0.63$ ), and MER (S: $0.600 \pm 0.315$ vs. R: $0.538 \pm 0.326 ; p=0.68$ ). While, based on numerical trends, MER-resistant isolates were the strongest biofilm producers, the ANOVA did not reveal significant differences $(p=0.067)$ between the biofilm production among antibiotic-resistant isolates. 


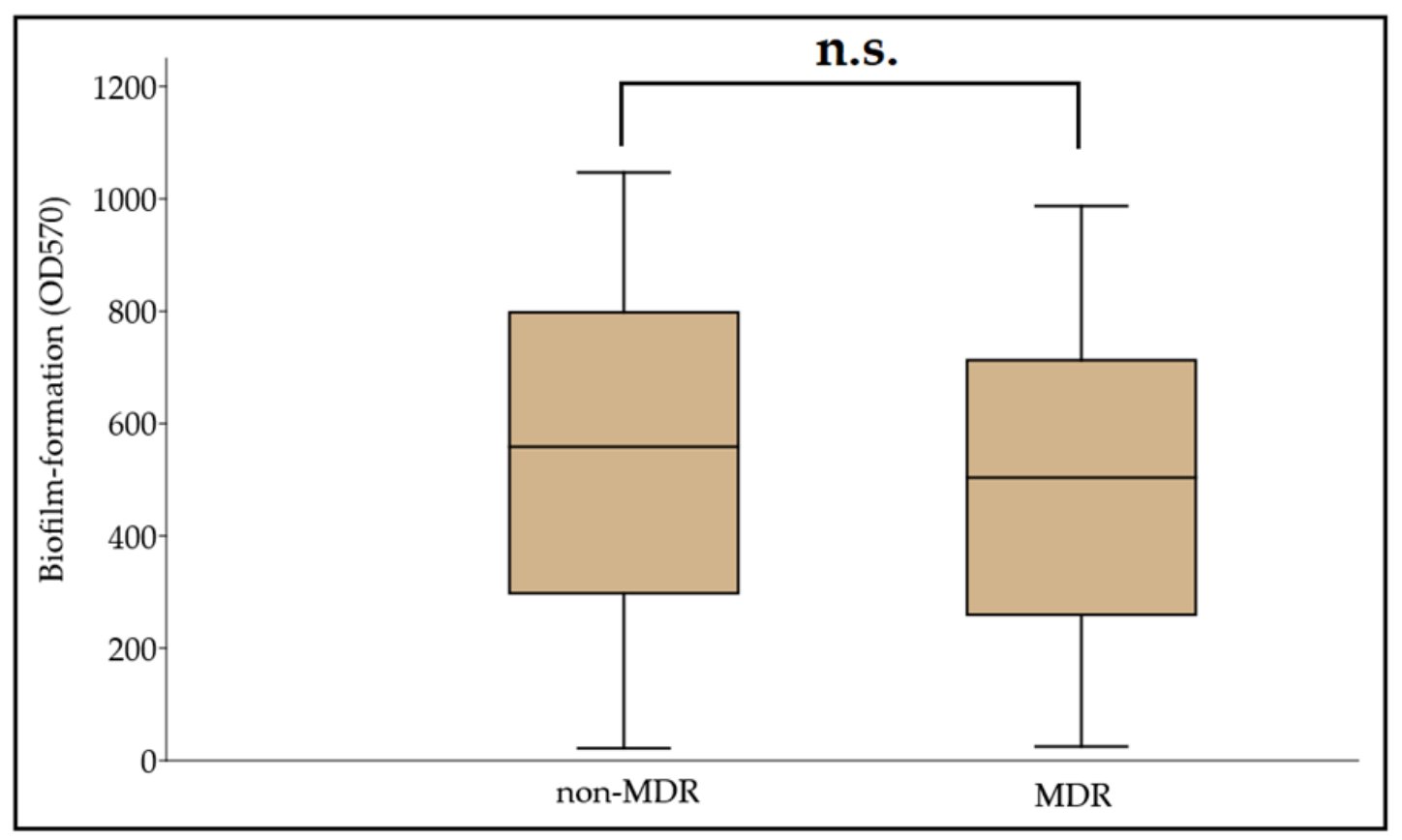

Figure 1. Comparison of biofilm-forming capacity among non-MDR and MDR A. baumannii isolates; n.s.: not significant $(p=0.072)$.

\section{Discussion}

In recent years, A. baumannii infections have presented with alarming rates of MDR; however, the global spread of $\mathrm{CR}-\mathrm{AB}$ was a critical hallmark of our fight against these pathogens, with their treatment emerging as an unmet medical need [33]. Carbapenemresistance may be mediated by a multitude of mechanisms (often co-occurring in these isolates), including outer membrane impermeability (often due to deficiency or loss of porins), overexpression of bacterial efflux pump systems, modifications in the cell wall or penicillin-binding proteins (PBPs), and through the production of $\beta$-lactamases (hyperproduction of AmpC cephalosporinases and carbapenemases) hydrolyzing these drugs [34]. In A. baumannii, Ambler Class D (OXA-type carbapenemases: the species possesses an intrinsic $\beta$-lactamase gene $\left(b l a_{\mathrm{OXA}-51-\text { like }}\right)$, which is often used for the identification of this species), and Class B (metallo- $\beta$-lactamases; MBLs) are the most prevalent, while Class A (serine $\beta$-lactamases) enzymes have also been described on occasion [35]. The relevance of these enzymes has increased in recent years (either alone or co-existing with OXA-type enzymes) and represents an important therapeutic conundrum, as there are currently no licensed inhibitors for their inhibition in clinical use [36]. As there is limited clinical experience with novel, innovative $\beta$-lactam-type drugs (e.g., ceftazidime/avibactam, imipenem/relebactam, meropenem/vaborbactam, and cefiderocol) for Acinetobacter infections, non- $\beta$-lactam-type drugs often need to be administered [37]. For a number of years, colistin was the drug-of-choice for CR-AB infections, due to the fact that Acinetobacter spp. are still largely susceptible to this drug; however, resistance rates have been steadily emerging in the recent years [38]. Other potentially useful drugs include tigecycline (where susceptibility data allow for it) and novel tetracycline derivatives (eravacycline and omadacycline), plazomicin and delafloxacin, or high-dose combinations of existing drugs, such as meropenem-colistin [26,37]. Nevertheless, it must be noted that many of the above-listed antimicrobials may have debilitating adverse events and may not be suitable for a wide array of patients. Based on the findings of a systematic review, acquiring the MDR status in Acinetobacter spp. is most often due to resistance to aminoglycosides and carbapenems, while XDR and PDR (pan-drug resistance) are often associated with resistance to colistin, tigecycline, and other ancillary antibiotics [38]. As a consequence of the Severe Acute Respiratory Syndrome Coronavirus-2 (SARS-CoV-2) pandemic and the associated disease 
(COVID-19), patients undergoing mechanical ventilation have soared; these patients are particularly at risk for nosocomial Acinetobacter spp. infections, due to the introduction of assisted ventilation and the preceding viral infection, which predisposes patients to bacterial superinfections. Rapid increases are expected in the resistance rates of Acinetobacter spp. and other bacterial pathogens, associated with the extensive and prophylactic use of antimicrobials in relation with COVID-19 [39].

As a part of our study, a large pool $(\mathrm{n}=309)$ of $A$. baumannii isolates from diverse geographical and clinical origins were subjected to phenotypic assays to characterize the possible correlation between biofilm-forming capacity and antimicrobial resistance in these isolates. In our pool of isolates, resistance rates were highest for the fluoroquinolones $(\sim 70 \%)$, while $>42 \%$ were classified as MDR. Over $20 \%$ of isolates were positive for carbapenemase production (although our methods may not have enough sensitivity to reliably detect all carbapenemases relevant in A. baumannii [29]), 9\% were positive for MBL production, while in $\sim 14 \%$, carbapenem nonsusceptibility was affected by efflux pump overexpression (based on the phenotypic tests applied). The levels of MDR in the isolates were similar to the MDR rates in Central Europe; however, carbapenem-resistance rates were $>50 \%$ [9]. Our results have highlighted that-based on susceptibility alone-colistin and tigecycline may still be optimal treatment choices for A. baumannii. A limitation of our study is that many additional antibiotics were not tested to establish the potential XDR or PDR status of the isolates [38].

The majority of isolates (62.14\% overall) were strong biofilm producers, both in the MDR and non-MDR groups; our tests conducted did not verify a significant difference in biofilm formation based on the susceptibilities of the relevant isolates to the tested antimicrobials. Based on the reports from the recent literature, the rate of biofilm formation in A. baumannii is around $75-100 \%$, which is similar to rates observed in NFGNB, and significantly higher than those of members of the Enterobacterales and Gram-positive bacteria (0-40\%); additionally, many studies have shown that environmental Acinetobacter isolates often produce more robust biofilms-probably with the aim of being resistant to desiccation-compared to isolates from clinical origins [17,40]. Zeighami et al. reported on the virulence characteristics of one hundred Acinetobacter spp. isolated from ICU patients: $91 \%$ of isolates were XDR, while $32 \%$ were resistant to all antibiotics tested, and the presence of class I and class II integrons was revealed in $67 \%$ and $10 \%$; all isolates $(100 \%)$ were either moderate or strong biofilm producers, with $100 \%, 98 \%, 95 \%, 92 \%$, and $81 \%$ with carriage of $c s u E, p g a B, e p s A, p t k, b f m S$, and the $\operatorname{omp} A$ biofilm-related genes, respectively [41]. The study of Hassan et al. involved $\mathrm{n}=74 \mathrm{~A}$. baumannii isolates (both from clinical material and from environmental origins, identified by the presence of the $b l a_{\text {OXA-51-like }}$ carbapenemase), which were assessed for the biofilm formation by three independent methods (CV tube method, CV tissue microtiter plates, and Congo Red Agar) in juxtaposition with their resistance. The majority of clinical isolates $(>90 \%)$ were resistant to ciprofloxacin, gentamicin, and carbapenems, although no carbapenemase genes were found during molecular testing. In addition, $64.86 \%$ of isolates were classified as moderate and strong biofilm producers; biofilm formation was stronger in isolates susceptible to ciprofloxacin, gentamicin, and imipenem/meropenem [42]. Qi et al. included $\mathrm{n}=272$ A. baumannii isolates from various general hospitals in China, which had resistance rates to ciprofloxacin, ceftazidime, trimethoprim/sulfamethoxazole, imipenem, amikacin, and polymyxin B of $65.8 \%, 61.0 \%, 59.9 \%, 59.2 \%, 46.0 \%$, and 3.7\%, respectively. In addition, $>72 \%$ classified as either MDR or XDR, and $23 \%$ were classified as strong biofilm producers, with non-MDR isolates producing a significantly more robust biofilm (this association was also verified for susceptibility to all individual antibiotics, apart from polymyxin B). Pulsefield gel electrophoresis (PFGE) analyses have revealed that the majority of nonsusceptible isolates-which were weak biofilm producers-belonged to the same eight main PFGE clusters [43].

Thummeepak et al. included $\mathrm{n}=225 \mathrm{~A}$. baumannii strains to perform phenotypic and molecular testing, during which $86.2 \%$ presented as MDR and $76.9 \%$ were biofilm produc- 
ers; the presence of virulence and biofilm-related genes (eps $A$, bap, omp $A$, and $b f m S$ ) showed significant association with the MDR phenotype in these isolates; additionally, a higher number of biofilm producers were detected among gentamicin-resistant $A$. baumannii [44] Greene et al. studied $\mathrm{n}=145$ clinical and environmental $A$. baumannii isolates for their biofilm-forming capacity: using cell death due to desiccation as an indicator, they found that non-MDR isolates were 2.7-times more susceptible to drying damage than the MDR isolates were [45]. Using A. baumannii clinical isolates and the ATCC 17978 reference strain as model organisms, Mayer et al. identified that operating quorum sensing (QS) systems are needed for functional motility and biofilm formation in vitro; their study highlighted that extracellular DNA is critical for the integrity of robust biofilms, in addition to showing that biofilm formation did not correlate with the antibiotic resistance of said isolates [46]. In addition, Selasi et al. noted that biofilm formation positively correlated with the expression level of many relevant virulence factors, such as surface proteins, pili, and the production of poly- $\beta-(1-6)-\mathrm{N}$-acetylglucosamine (PNAG) and acyl-homoserine lactone (AHL) signal molecules [47].

Several authors have aimed to propose a definite genetic link between the propensity to form biofilms and antimicrobial resistance in A. baumannii [17]. In the study of Aziz et al., A. baumannii isolates-which carried the extended spectrum $\beta$-lactamase bla $a_{\text {PER-1 }}$ - produced a significantly more robust biofilm than the gene-deficient bacteria; their results showed that $b l a_{\text {PER-1 }}$-positive isolates more efficiently adhered to epithelial cells in in vitro settings, and this attribute may be relevant in the early stages of biofilm production [48]. On the other hand, Gallant et al. showed contrasting findings for P. aeruginosa, where isolates presenting with and expressing the $\beta$-lactamase gene $b a_{\mathrm{TEM}-1}$ had limited ability to form biofilms, which was suggested to be a consequence of the low adhesive potential of these strains [49]. The biofilm-associated protein (Bap) is a cell surface protein in Acinetobacter spp.--homologous with the Bap protein found in Staphylococcus spp.-which has important roles to surface attachment and biofilm maturation. In the context of antibiotic resistance, it has been suggested that interactions of Bap and the outer membrane protein OmpA, which functions as the major porin A. baumannii, may have relevance in biofilm-forming capacity; namely, porin-deficient strains may show less pronounced biofilm production [18]. Our study emphasizes the need for additional experiments to assess the role biofilms have in the pathogenesis of A. baumannii infections.

Author Contributions: M.G., M.G.D. and S.Z. conceived and designed the study. M.G.D., M.G., V.M., P.C., S.Z. and K.B. were involved in the collection of isolates and in performing the experiments. M.G.D., M.G., Á.L.N., V.M. and A.S. provided resources. M.G.D., M.M., P.C. and K.B. supervised the project. M.G. and M.G.D. wrote the initial draft of the manuscript. All authors have read and agreed to the published version of the manuscript.

Funding: M.G. was supported by the János Bolyai Research Scholarship (BO/00144/20/5) of the Hungarian Academy of Sciences. The research was supported by the ÚNKP-21-5-540-SZTE New National Excellence Program of the Ministry for Innovation and Technology from the source of the National Research. Development and Innovation Fund. M.G. would also like to acknowledge the support of ESCMID's “30 under 30" Award.

Institutional Review Board Statement: The study was conducted in accordance with the Declaration of Helsinki and national and institutional ethical standards. Ethical approval for the study protocol was obtained from the Human Institutional and Regional Biomedical Research Ethics Committee, University of Szeged (registration number: 140/2021-SZTE [5019]).

Informed Consent Statement: Not applicable.

Data Availability Statement: All data generated during the study are presented in this paper.

Conflicts of Interest: The authors declare no conflict of interest, monetary or otherwise. The authors alone are responsible for the content and writing of this article. 


\section{References}

1. Cannas, S.; Usai, D.; Pinna, A.; Benvenuti, S.; Tardugno, R.; Donadu, M.; Zanetti, S.; Kaliamurthy, J.; Molicotti, P. Essential oils in ocular pathology: An experimental study. J. Infect. Dev. Ctries 2015, 9, 650-654. [CrossRef] [PubMed]

2. Zhang, H.Z.; Zhanh, J.S.; Qiao, L. The Acinetobacter baumannii group: A systemic review. World J. Emerg. Med. 2013, 4, 169-174. [CrossRef]

3. Morris, F.C.; Dexter, C.; Kostoulias, X.; Uddin, M.I.; Peleg, A. The mechanisms of disease caused by Acinetobacter baumannii. Front Microbiol. 2019, 10, e1601. [CrossRef] [PubMed]

4. Chou, C.H.; Lai, Y.R.; Chi, C.Y.; Ho, M.W.; Chen, C.L.; Liao, W.C.; Ho, C.H.; Chen, Y.A.; Chen, C.Y.; Lin, Y.T.; et al. Long-term surveillance of antibiotic prescriptions and the prevalence of antimicrobial resistance in non-fermenting Gram-negative bacilli. Microorganisms 2020, 8, 397. [CrossRef] [PubMed]

5. Pinna, A.; Donadu, M.G.; Usai, D.; Dore, S.; Boscia, F.; Zanetti, S. In Vitro antimicrobial activity of a new ophthalmic solution containing Hexamidine Diisethionate 0.05\% (Keratosept). Cornea 2020, 39, 1415-1418. [CrossRef] [PubMed]

6. Wong, D.; Nielsen, T.B.; Bonomo, R.A.; Pantapalangkoor, P.; Luna, B.; Spellberg, B. Clinical and Pathophysiological Overview of Acinetobacter Infections: A Century of Challenges. Clin. Microbiol. Rev. 2017, 30, 409-447. [CrossRef]

7. Ma, C.; McClean, S. Mapping Global Prevalence of Acinetobacter baumannii and Recent Vaccine Development to Tackle It. Vaccines 2021, 9, 570. [CrossRef]

8. Pascale, R.; Corcione, S.; Bussini, L.; Pancaldi, L.; Giacobbe, R.D.; Ambretti, S.; Lupia, T.; Costa, C.; Marchese, A.; De Rosa, F.G.; et al. Non-fermentative gram-negative bloodstream infection in northern Italy: A multicenter cohort study. BMC Infect. Dis. 2021, 21, e806. [CrossRef] [PubMed]

9. Lim, S.M.S.; Abidin, A.Z.; Liew, S.M.; Roberts, J.A.; Sime, F.B. The global prevalence of multidrug-resistance among Acinetobacter baumannii causing hospital-acquired and ventilator-associated pneumonia and its associated mortality: A systematic review and meta-analysis. J. Infect. 2019, 79, 593-600.

10. Da Cunda, P.; Iribarnegaray, V.; Papa-Ezdra, R.; Bado, I.; González, M.J.; Zunino, P.; Vignoli, R.; Scavone, P. Characterization of the Different Stages of Biofilm Formation and Antibiotic Susceptibility in a Clinical Acinetobacter baumannii Strain. Microb. Drug Res. 2020, 26, 569-575. [CrossRef]

11. Amin, M.; Navidifar, T.; Shooshtari, F.S.; Goodarzi, H. Association of the genes encoding Metallo- $\beta$-Lactamase with the presence of integrons among multidrug-resistant clinical isolates of Acinetobacter baumannii. Infect. Drug Res. 2019, 12, 1171-1180. [CrossRef]

12. World Health Organisation (WHO). Global Priority List of Antibiotic-Resistant Bacteria to Guide Research, Discovery, and Development of New Antibiotics; World Health Organisation: Geneva, Switzerland, 2017; pp. 1-7.

13. Maurice, N.M.; Bedi, B.; Sadikot, R.T. Pseudomonas aeruginosa Biofilms: Host Response and Clinical Implications in Lung Infections. Am. J. Respir. Cell Mol. Biol. 2018, 58, 428-439. [CrossRef] [PubMed]

14. Cifou, O.; Rojo-Molinero, E.; Macia, M.D.; Oliver, A. Antibiotic treatment of biofilm infections. APMIS 2017, 125, 304-319. [CrossRef] [PubMed]

15. Jamal, M.; Ahmad, W.; Andleeb, S.; Jalil, F.; Imran, M.; Nawaz, M.A.; Hussain, T.; Ali, M.; Rafiq, M.; Kamil, M.A. Bacterial biofilm and associated infections. J. Chin. Med. Assoc. 2018, 81, 7-11. [CrossRef] [PubMed]

16. Azeredo, J.; Azevedo, N.F.; Briandet, R.; Cerca, N.; Coenye, T.; Costa, A.R.; Desvaux, M.; Di Bonaventura, G.; Hébraud, M.; Jaglic, Z.; et al. Critical review on biofilm methods. Crit. Rev. Microbiol. 2017, 43, 313-351. [CrossRef] [PubMed]

17. Pompilio, A.; Scribano, D.; Sarshar, M.; Di Bonaventura, G.; Palamara, A.T.; Ambrosi, C. Gram-Negative Bacteria Holding Together in a Biofilm: The Acinetobacter baumannii Way. Microorganisms 2021, 9, 1353. [CrossRef] [PubMed]

18. Tahaei, S.A.S.; Stájer, A.; Barrak, I.; Ostorházi, E.; Szabó, D.; Gajdács, M. Correlation Between Biofilm-Formation and the Antibiotic Resistant Phenotype in Staphylococcus aureus Isolates: A Laboratory-Based Study in Hungary and a Review of the Literature. Infect. Drug Res. 2021, 14, 1155-1168. [CrossRef] [PubMed]

19. Cepas, V.; López, Y.; Munoz, E.; Rolo, D.; Ardanuy, C.; Martí, M.; Xercavins, M.; Horcajada, J.P.; Bosch, J.; Soto, S.M. Relationship Between Biofilm Formation and Antimicrobial Resistance in Gram-Negative Bacteria. Microb. Drug Res. 2019, $25,72-83$. [CrossRef] [PubMed]

20. Avila-Novoa, M.G.; Solís-Velázquez, O.A.; Rangel-López, D.E.; González-Gómez, J.P.; Guerrero-Medin, P.J.; Gutiérez-Lomelí, M. Biofilm Formation and Detection of Fluoroquinolone- and Carbapenem-Resistant Genes in Multidrug-Resistant Acinetobacter baumannii. Can. J. Infect. Dis. Med. Microbiol. 2019, 2019, e3454907. [CrossRef]

21. Ryu, S.Y.; Baek, W.K.; Kim, H.A. Association of biofilm production with colonization among clinical isolates of Acinetobacter baumannii. Korean J. Intern. Med. 2017, 32, 345-351. [CrossRef]

22. Gajdács, M.; Bátori, Z.; Ábrók, M.; Lázár, A.; Burián, K. Characterization of Resistance in Gram-Negative Urinary Isolates Using Existing and Novel Indicators of Clinical Relevance: A 10-Year Data Analysis. Life 2020, 11, 16. [CrossRef]

23. Schubert, S.; Kostrzewa, M. MALDI-TOF MS in the microbiology laboratory: Current trends. Curr. Issues Mol. Biol. 2017, 23, 17-20. [CrossRef] [PubMed]

24. Sadat, A.; El-Sherbiny, H.; Zakaria, A.; Ramadan, H.; Awad, A. Prevalence, antibiogram and virulence characterization of Vibrio isolates from fish and shellfish in Egypt: A possible zoonotic hazard to humans. J. Appl. Microbiol. 2021, 131, 485-498. [CrossRef]

25. European Committee on Antimicrobial Susceptibility Testing (EUCAST). Clinical Breakpoints and Dosing. Available online: https:/ / www.eucast.org/clinical_breakpoints / (accessed on 5 August 2021). 
26. Navidifar, T.; Amin, M.; Rashno, M. Effects of sub-inhibitory concentrations of meropenem and tigecycline on the expression of genes regulating pili, efflux pumps and virulence factors involved in biofilm formation by Acinetobacter baumannii. Infect. Drug Res. 2019, 12, 1099-1111. [CrossRef] [PubMed]

27. Magiorakos, A.P.; Srinivasan, A.; Carey, R.B.; Carmeli, Y.; Falagas, M.E.; Giske, C.G.; Paterson, D.L. Multidrug-resistant, extensively drug-resistant and pandrug-resistant bacteria: An international expert proposal for interim standard definitions for acquired resistance. Clin. Microbiol. Infect. 2012, 18, 268-281. [CrossRef]

28. Kichibiro, T.; Komatsu, M.; Yamasaki, K.; Nakamura, T.; Nishio, H.; Kimura, K.; Niki, M.; Kida, N.; Ohama, M.; Fukuda, N.; et al. Comparison of the performance of three carbapenem inactivation methods for the detection of carbapenemase-producing Gram-negative bacilli. J. Infect. Chemother. 2021, 27, 1634-1638. [CrossRef] [PubMed]

29. Abouelfetouh, A.; Torky, A.S.; Aboulmagd, E. Phenotypic and genotypic characterization of carbapenem-resistant Acinetobacter baumannii isolates from Egypt. Antimicrob. Res. Infect. Control 2019, 8, e185. [CrossRef] [PubMed]

30. Salehi, B.; Ghalavand, Z.; Yadegar, A.; Eslami, G. Characteristics and diversity of mutations in regulatory genes of resistancenodulation-cell division efflux pumps in association with drug-resistant clinical isolates of Acinetobacter baumannii. Antimicrob. Res. Infect. Control 2021, 10, e53. [CrossRef] [PubMed]

31. Ramos-Vivas, J.; Chapartegui-González, I.; Fernández-Martínez, M.; González-Rico, C.; Fortún, J.; Escudero, R.; Marco, F.; Linares, L.; Montejo, M.; Aranzamendi, M.; et al. Biofilm formation by multidrug resistant Enterobacteriaceae strains isolated from solid organ transplant recipients. Sci. Rep. 2019, 9, e8928. [CrossRef]

32. Stepanovic, S.; Vukovic, D.; Hola, V.; Di Bonaventura, G.; Djukovic, S.; Cirkovic, I.; Ruzicka, F. Quantification of biofilm in microtiter plates: Overview of testing conditions and practical recommendations for assessment of biofilm production by staphylococci. APMIS 2007, 115, 891-899. [CrossRef]

33. Isler, B.; Doi, Y.; Bonomo, R.A.; Paterson, D.L. New treatment options against carbapenem-resistant Acinetobacter baumannii infections. Antimicrob. Agents Chemother. 2019, 63, e01110-e01118. [CrossRef] [PubMed]

34. Tarashi, S.; Goodarzi, H.; Erfanimanesh, S.; Pormohammad, A.; Hashemi, A. Phenotypic and molecular detection of metallo-betalactamase genes among imipenem resistant Pseudomonas aeruginosa and Acinetobacter baumannii strains isolated from patients with burn injuries. Arch. Clin. Infect. Dis. 2016, 11, e39036. [CrossRef]

35. Nordmann, P.; Poirel, L. Epidemiology and diagnostics of carbapenem resistance in Gram-negative bacteria. Clin. Infect. Dis. 2019, 69, S521-S528. [CrossRef]

36. Gheorghe, I.; Barbu, I.C.; Surleac, M.; Sarbu, I.; Popa, L.I.; Paraschiv, S.; Feng, Y.; Lazar, V.; Chifiriuc, M.C.; Otelea, D.; et al. Subtypes, resistance and virulence platforms in extended-drug resistant Acinetobacter baumannii Romanian isolates. Sci. Rep. 2021, 11, e13288. [CrossRef] [PubMed]

37. Paterson, D.L.; Isler, B.; Stewart, A. New treatment options for multiresistant Gram-negatives. Curr. Opin. Infect. Dis. 2020, 33, 214-223. [CrossRef]

38. Banafsheh, D.M.; Hesan, A.; Hossein, K.; Afsaneh, K. Antimicrobial categories in describing multidrug resistance, extensive drug resistance and pan-drug resistance in Pseudomonas aeruginosa and Acinetobacter baumannii: A systematic review. Rev. Med. Microbiol. 2021, 32, 6-11.

39. Rangel, R.; Chagas, T.P.G.; De-Simone, S.G. Acinetobacter baumannii Infections in Times of COVID-19 Pandemic. Pathogens 2021, 10, 1006. [CrossRef]

40. Sarshar, M.; Behzadi, P.; Scribano, D.; Palama, A.T.; Ambrosi, C. Acinetobacter baumannii: An Ancient Commensal with Weapons of a Pathogen. Pathogens 2021, 10, 387. [CrossRef] [PubMed]

41. Zeighami, H.; Valadkhani, F.; Shapouri, R.; Samadi, E.; Haghi, F. Virulence characteristics of multidrug resistant biofilm forming Acinetobacter baumannii isolated from intensive care unit patients. BMC Infect. Dis. 2019, 19, e629. [CrossRef]

42. Hassan, P.A.; Kihder, A.K. Correlation of biofilm formation and antibiotic resistance among clinical and soil isolates of Acinetobacter baumannii in Iraq. Acta Microbiol. Immunol. Hung. 2020, 67, 161-170. [CrossRef] [PubMed]

43. Qi, L.; Li, H.; Zhang, C.; Liang, B.; Li, J.; Wang, L.; Du, X.; Liu, X.; Qiu, S.; Song, H. Relationship between antibiotic resistance, biofilm formation, and biofilm-specific resistance in Acinetobacter baumannii. Front. Microbiol. 2016, 7, e483. [CrossRef] [PubMed]

44. Thummeepak, R.; Kongthai, P.; Leungtongkam, U.; Sittihisak, S. Distribution of virulence genes involved in biofilm formation in multi-drug resistant Acinetobacter baumannii clinical isolates. Int. Microbiol. 2016, 19, 121-129. [PubMed]

45. Greene, C.; Vadlamudi, G.; Newton, D.; Foxman, B.; Xi, C. The influence of biofilm formation and multidrug resistance on environmental survival of clinical and environmental isolates of Acinetobacter baumannii. Am. J. Infect. Control 2016, 44, e65-e71. [CrossRef] [PubMed]

46. Mayer, C.; Muras, A.; Parga, A.; Romero, M.; Rumbo-Feal, S.; Poza, M.; Ramos-Vivas, J.; Otero, A. Controlling Surface Associated Motility and Biofilm Formation in Acinetobacter baumannii ATCC-17978TM. Front. Microbiol. 2020, 11, e565548. [CrossRef]

47. Selasi, G.N.; Nicholas, A.; Jeon, H.; Na, S.H.; Kwon, H.; Kim, J.Y.; Heo, S.T.; Oh, H.M.; Lee, J.C. Differences in biofilm mass, expression of biofilm-associated genes, and resistance to desiccation between epidemic and sporadic clones of carbapenemresistant Acinetobacter baumannii sequence type 191. PLoS ONE 2016, 11, e0162576. [CrossRef] [PubMed] 
48. Azizi, O.; Shahcheraghi, F.; Salimizand, H.; Modarresi, F.; Shakibaie, M.R.; Mansouri, S.; Ramazanzadeh, R.; Badmasti, F.; Nikbin, V. Molecular Analysis and Expression of bap Gene in Biofilm-Forming Multi-Drug-Resistant Acinetobacter baumannii. Rep. Biochem. Mol. Biol. 2016, 5, 62-72. [PubMed]

49. Gallant, C.V.; Daniels, C.; Leung, J.M.; Ghosh, A.S.; Young, K.D.; Kotra, L.P.; Burrows, L.L. Common $\beta$-lactamases inhibit bacterial biofilm formation. Mol. Microbiol. 2005, 58, 1012-1024. [CrossRef] [PubMed] 